\title{
A construction of Abelian non-cyclic orbit code*
}

\author{
Joan-Josep Climent, Verónica Requena, Xaro Soler-Escrivà \\ Departament de Matemàtiques, Universitat d'Alacant, \\ Ap. Correus 99, E-03080, Alacant, Spain
}

April 17, 2018

\begin{abstract}
A constant dimension code consists of a set of $k$-dimensional subspaces of $\mathbb{F}_{q}^{n}$, where $\mathbb{F}_{q}$ is a finite field of $q$ elements. Orbit codes are constant dimension codes which are defined as orbits under the action of a subgroup of the general linear group on the set of all $k$-dimensional subspaces of $\mathbb{F}_{q}^{n}$. If the acting group is Abelian, we call the corresponding orbit code Abelian orbit code. In this paper we present a construction of an Abelian non-cyclic orbit code for which we compute its cardinality and its minimum subspace distance. Our code is a partial spread and consequently its minimum subspace distance is maximal.
\end{abstract}

Keywords: Random linear network coding, subspace codes, Grassmannian, group action, general linear group, Abelian group.

\section{Introduction}

Random linear network coding, introduced by Ahlswede, Cai, Li and Yeung [1], is a powerful tool for disseminating information in networks with unknown or changing topology [13]. The main feature of these networks is that the packets of information are vectors over a finite field $\mathbb{F}_{q}$ and each internal node of the network transmits random linear combinations of the received packets to adjacent nodes. However, due to the linear combination of the nodes, this method is highly susceptible to error propagation. Trying to solve this problem, Kötter and Kschischang [14] proposed a mathematical description of random network coding by considering messages as subspaces of some fixed vector space $\mathbb{F}_{q}^{n}$. This algebraic approach captures the main feature of the network flow: the linear combination of the packets. Now, codewords are simply subspaces of $\mathbb{F}_{q}^{n}$ and a code is a collection of subspaces.

The study of subspace codes has led to many papers in recent years (see, for example, $[3,4,6,9,10$, $12,21,22,23,24,25]$ and the references therein). Most of these authors focus on the study of constant dimension codes; that is, subspace codes in which all subspaces have the same dimension.

The paper at hand is most closely related to references [2, 8, 9, 19, 20, 24, 25, 26]. All these papers deal with orbit codes, which are constant dimension codes that arise as an orbit of a subspace in $\mathbb{F}_{q}^{n}$ under the action of a subgroup of the general linear group. Orbit codes were first introduced in the area of network coding in [25], where the authors showed how the Reed-Solomon type codes introduced in [14] as well as the spread codes described in [18], can be seen as special instances of orbit codes. Orbit codes can be classified according to the groups used to construct the orbit. Most of the previous references deal with cyclic subgroups of the general linear group. Our aim is to go a bit further. We are interested in Abelian non-cyclic orbit codes; that is, orbit codes which are generated by Abelian non-cyclic subgroups

\footnotetext{
${ }^{*}$ This work was partially supported by Spanish grants AICO/2017/128 of the Generalitat Valenciana and VIGROB287 of the Universitat d'Alacant
} 
of the general linear group. Since Abelian groups are direct product of cyclic subgroups, it seems a natural extension of the research on cyclic orbit codes. As far as we know, this type of orbit codes is new in the literature of orbit codes. We wonder if this generalization leads to some advantages, in terms of a wider set of parameters for code designs, for instance. As a first step in this research, we present a construction of an Abelian non-cyclic orbit code for which we compute its cardinality and its minimum subspace distance. For this, we consider two commuting upper triangular matrices as generators of our Abelian subgroup. To the best of our knowledge, this is the first time that this type of matrices has been used for constructing orbit codes. The resulting construction is an optimal code with respect to the subspace distance for a given set of parameters $(n, k)$, that is, our code is partial spread [11].

The rest of the paper is structured as follows: In Section 2 we give some preliminaries, first about random linear network coding and orbit codes and then a result about upper triangular matrices. The main body of the paper is Section 3, where the whole construction of our Abelian non-cyclic orbit code is given. We finish the paper with the conclusions of our work and some open questions in Section 4 .

\section{Preliminaries}

Let $\mathbb{F}_{q}$ be the finite field of $q$ elements, where $q$ is a prime power. For any positive integer $n$, it is well-known that $\mathcal{P}_{q}(n)$, the set of all vector subspaces of $\mathbb{F}_{q}^{n}$, forms a metric space with respect to the subspace distance defined by (see [14])

$$
d(\mathcal{U}, \mathcal{V})=\operatorname{dim}(\mathcal{U}+\mathcal{V})-\operatorname{dim}(\mathcal{U} \cap \mathcal{V})=\operatorname{dim}(\mathcal{U})+\operatorname{dim}(\mathcal{V})-2 \operatorname{dim}(\mathcal{U} \cap \mathcal{V}), \quad \text { for all } \mathcal{U}, \mathcal{V} \in \mathcal{P}_{q}(n)
$$

For any integer $k$, where $0 \leq k \leq n$, the set of all $k$-dimensional vector subspaces of $\mathbb{F}_{q}^{n}$ is called Grassmann variety (or simply Grassmannian) and is denoted by $\mathcal{G}_{q}(k, n)$. Obviously, $\mathcal{P}_{q}(n)=$ $\cup_{k=0}^{n} \mathcal{G}_{q}(k, n)$.

A subspace code of length $n$ is a nonempty subset $\mathfrak{C}$ of $\mathcal{P}_{q}(n)$; a codeword of such a code is a vector subspace of $\mathfrak{C}$. The minimum distance [14] of a subspace code $\mathfrak{C} \subseteq \mathcal{P}_{q}(n)$ is defined as

$$
d(\mathfrak{C})=\min \{d(\mathcal{U}, \mathcal{V}) \mid \mathcal{U}, \mathcal{V} \in \mathfrak{C}, \mathcal{U} \neq \mathcal{V}\}
$$

The code $\mathfrak{C}$ is called equidistant (see [12]) if $d(\mathcal{U}, \mathcal{V})=d(\mathfrak{C})$ for all $\mathcal{U}, \mathcal{V} \in \mathfrak{C}$ with $\mathcal{U} \neq \mathcal{V}$. Moreover, the code $\mathfrak{C}$ is called a partial spread of $\mathbb{F}_{q}^{n}$ (see [11]) if $\mathcal{U} \cap \mathcal{V}=\{0\}$, for all $\mathcal{U}, \mathcal{V} \in \mathfrak{C}$ with $\mathcal{U} \neq \mathcal{V}$.

We call $\mathfrak{C}$ a constant dimension code (see [14]) if all codewords of $\mathfrak{C}$ have the same dimension; that is, if $\mathfrak{C} \subseteq \mathcal{G}_{q}(k, n)$ for some $k$. We may assume, without loss of generality, that $k \leq n / 2$; otherwise, we can consider the complementary code (or dual code)

$$
\mathfrak{C}^{\perp}=\left\{\mathcal{U}^{\perp} \subseteq \mathcal{G}_{q}(n-k, n) \mid \mathcal{U} \in \mathfrak{C}\right\}
$$

obtained from the orthogonal subspaces of the codewords of $\mathfrak{C}$, which has the same length, cardinality and minimum distance (see [14]).

In order to represent a $k$-dimensional subspace $\mathcal{U} \in \mathcal{G}_{q}(k, n)$, we use a generator matrix $U \in \mathbb{F}_{q}^{k \times n}$ whose rows form a basis of $\mathcal{U}$; that is, $U$ is a full-rank matrix and $\mathcal{U}=\operatorname{rowspace}(U)=\left\{\boldsymbol{x} U \mid \boldsymbol{x} \in \mathbb{F}_{q}^{k}\right\}$.

Note that in $\mathcal{G}_{q}(k, n)$ the subspace distance of expression (1) is given by

$$
d(\mathcal{U}, \mathcal{V})=2(k-\operatorname{dim}(\mathcal{U} \cap \mathcal{V}))=2 \operatorname{rank}\left[\begin{array}{l}
U \\
V
\end{array}\right]-2 k \leq 2 k
$$

for any $\mathcal{U}, \mathcal{V} \in \mathcal{G}_{q}(k, n)$ and some respective generator matrices $U, V \in \mathbb{F}_{q}^{k \times n}$.

In particular, if a constant dimension code $\mathfrak{C} \subseteq \mathcal{G}_{q}(k, n)$ is a partial spread then $\mathfrak{C}$ is equidistant and its minimum distance is maximal, that is, $2 k$.

We focus on constant dimension codes arising from group actions, which are simply called orbit codes and which were introduced in [25] (see also [24]). 
The general linear group of degree $n$, denoted by $\mathrm{GL}_{n}$, is the set of all invertible $n \times n$ matrices with entries in $\mathbb{F}_{q}$. Given a full-rank matrix $U \in \mathbb{F}_{q}^{k \times n}$, its rowspace $\mathcal{U}=$ rowspace $(U) \in \mathcal{G}_{q}(k, n)$, and $A \in \mathrm{GL}_{n}$, we define

$$
\mathcal{U} \cdot A=\operatorname{rowspace}(U A) .
$$

Provided that this operation is independent from the representation of $\mathcal{U}$ (see [25]) and since any invertible matrix maps vector subspaces to vector subspaces of the same dimension, we obtain a group action (from the right) on the Grassmann variety:

$$
\begin{array}{cl}
\mathcal{G}_{q}(k, n) \times \mathrm{GL}_{n} & \longrightarrow \mathcal{G}_{q}(k, n) \\
(\mathcal{U}, A) & \mapsto \mathcal{U} \cdot A
\end{array}
$$

It is well-known that two $k$-dimensional subspaces can be mapped onto each other by an invertible matrix. Therefore, the orbit of any $k$-dimensional subspace $\mathcal{U}$ under the action of $\mathrm{GL}_{n}$ is the whole set $\mathcal{G}_{q}(k, n)$. That is, $\mathrm{GL}_{n}$ acts transitively on $\mathcal{G}_{q}(k, n)$.

Now, let $\mathbf{G}$ be a subgroup of $\mathrm{GL}_{n}$ and consider the action of $\mathbf{G}$ on $\mathcal{G}_{q}(k, n)$. Then the orbit of a subspace $\mathcal{U} \in \mathcal{G}_{q}(k, n)$ under the action of $\mathbf{G}$ is

$$
\mathfrak{C}=\{\mathcal{U} \cdot A \mid A \in \mathbf{G}\},
$$

which is called the orbit code generated by the action of $\mathbf{G}$ on $\mathcal{U}$ (see [25]). The stabilizer of $\mathcal{U}$ under this action is

$$
\operatorname{Stab}_{\mathbf{G}}(\mathcal{U})=\{A \in \mathbf{G} \mid \mathcal{U} \cdot A=\mathcal{U}\}=\mathbf{G} \cap \operatorname{Stab}_{\mathrm{GL}_{n}}(\mathcal{U})
$$

and the size $|\mathfrak{C}|$ of the orbit code $\mathfrak{C}$ is

$$
|\mathfrak{C}|=\frac{|\mathbf{G}|}{\left|\operatorname{Stab}_{\mathbf{G}}(\mathcal{U})\right|}
$$

Moreover (see [25, Proposition 8]), the subspace distance is $\mathrm{GL}_{n}$-invariant, that is,

$$
d(\mathcal{U}, \mathcal{V})=d(\mathcal{U} \cdot A, \mathcal{V} \cdot A), \quad \text { for all } A \in \mathrm{GL}_{n} ;
$$

this property allows us to compute the minimum distance of $\mathfrak{C}$ in a simple manner:

$$
d(\mathfrak{C})=\min \left\{d(\mathcal{U}, \mathcal{U} \cdot A) \mid A \in \mathbf{G} \backslash \operatorname{Stab}_{\mathbf{G}}(\mathcal{U})\right\} .
$$

The following lemma on block upper triangular matrices will be useful for the rest of the paper. The proof is straightforward and we omit it.

Lemma 1: Assume that $A \in \mathbb{F}_{q}^{r \times r}, B \in \mathbb{F}_{q}^{s \times s}$, and $P \in \mathbb{F}_{q}^{r \times s}$ and consider the block upper triangular matrix

$$
M=\left[\begin{array}{ll}
A & P \\
O & B
\end{array}\right]
$$

where $O$ denotes the zero matrix of the appropriate size. If $h$ is a nonnegative integer, then

$$
M^{h}=\left[\begin{array}{cc}
A^{h} & \Psi_{h}(A, B, P) \\
O & B^{h}
\end{array}\right]
$$

where

$$
\Psi_{h}(A, B, P)= \begin{cases}O, & \text { if } h=0, \\ \sum_{i=1}^{h} A^{h-i} P B^{i-1}, & \text { if } h \geq 1 .\end{cases}
$$

Moreover, if $i$ and $j$ are nonnegative integers, then

$$
\Psi_{i+j}(A, B, P)=A^{i} \Psi_{j}(A, B, P)+\Psi_{i}(A, B, P) B^{j} .
$$


Our aim is to construct an orbit code $\mathfrak{C}$ by considering the orbit of $\mathcal{U}_{k}$, the standard $k$-dimensional vector subspace; that is, the subspace generated by the first $k$ unit vectors of $\mathbb{F}_{q}^{n}$, under the action of a concrete subgroup $\mathbf{G}$ of $\mathrm{GL}_{n}$. If $I_{k}$ denotes the identity matrix of size $k \times k$, then $\mathcal{U}_{k}=$ rowspace $\left(\left[I_{k} O\right]\right)$.

The reason why we have chosen $\mathcal{U}_{k}$ is the following: given a vector space $\mathcal{U} \in \mathcal{G}_{q}(k, n)$ and an orbit code $\mathfrak{C}=\{\mathcal{U} \cdot A \mid A \in \mathbf{G}\}$ generated by the action of a subgroup $\mathbf{G}$ of $\mathrm{GL}_{n}$, there always exists a subgroup $\mathbf{G}^{\prime}$ of $\mathrm{GL}_{n}$ which is conjugate with $\mathbf{G}$, and such that the orbit code $\mathfrak{C}^{\prime}=\left\{\mathcal{U}_{k} \cdot B \mid B \in \mathbf{G}^{\prime}\right\}$ has the same cardinality, the same length, and the same minimum distance than $\mathfrak{C}$ (see [25, Proposition 11]).

In order to choose our group $\mathbf{G}$, we have taken into account different factors.

First, Lemma 1 provides an easy tool for working with block upper triangular matrices. Moreover, the stabilizer of $\mathcal{U}_{k}$ in $\mathrm{GL}_{n}$ is composed of block lower triangular invertible matrices. In fact, it can be readily verified that

$$
\operatorname{Stab}_{\mathrm{GL}_{n}}\left(\mathcal{U}_{k}\right)=\left\{\left[\begin{array}{cc}
A & O \\
Q & B
\end{array}\right] \mid A \in \mathrm{GL}_{k}, B \in \mathrm{GL}_{n-k} \text { and } Q \in \mathbb{F}_{q}^{(n-k) \times k}\right\} .
$$

Therefore, it turns out that taking $\mathbf{G}$ as a subgroup of block upper triangular invertible matrices, the stabilizer of $\mathcal{U}_{k}$ in $\mathbf{G}$ is readily described by block diagonal invertible matrices (see expression (5) below). Concretely, assume that $\mathbf{G}$ is a subgroup of block upper triangular matrices of $\mathrm{GL}_{n}$ and consider the following block partition of any matrix

$$
G=\left[\begin{array}{cc}
G_{11} & G_{12} \\
O & G_{22}
\end{array}\right] \in \mathbf{G}
$$

where $G_{11} \in \mathrm{GL}_{k}, G_{12} \in \mathbb{F}_{q}^{k \times(n-k)}, G_{22} \in \mathrm{GL}_{n-k}$, and $O$ denotes the zero matrix of the appropriate size. Since $\operatorname{Stab}_{\mathbf{G}}\left(\mathcal{U}_{k}\right)=\mathbf{G} \cap \operatorname{Stab}_{\mathrm{GL}_{n}}\left(\mathcal{U}_{k}\right)$, using the previous partition of matrices of $\mathbf{G}$ given by expression (4), the stabilizer of $\mathcal{U}_{k}$ in $\mathbf{G}$ can be written as

$$
\operatorname{Stab}_{\mathbf{G}}\left(\mathcal{U}_{k}\right)=\left\{\left[\begin{array}{cc}
G_{11} & G_{12} \\
O & G_{22}
\end{array}\right] \in \mathbf{G} \mid G_{12}=O\right\} .
$$

Secondly, taking $\mathbf{G}$ as a subgroup of block upper triangular matrices of $\mathrm{GL}_{n}$ provides us an easy upper bound on the minimum distance of the orbit code $\mathfrak{C}$. In fact, since the orbit code $\mathfrak{C}$ can be written as

$$
\mathfrak{C}=\left\{\mathcal{U}_{k} \cdot G \mid G \in \mathbf{G}\right\}=\left\{\operatorname{rowspace}\left(\left[\begin{array}{ll}
G_{11} & G_{12}
\end{array}\right]\right) \mid\left[\begin{array}{cc}
G_{11} & G_{12} \\
O & G_{22}
\end{array}\right] \in \mathbf{G}\right\},
$$

according to expressions (2) and (4), for any $G \in \mathbf{G}$ we have

$$
d\left(\mathcal{U}_{k}, \mathcal{U}_{k} \cdot G\right)=2 \operatorname{rank}\left[\begin{array}{cc}
I_{k} & O \\
G_{11} & G_{12}
\end{array}\right]-2 k=2 \operatorname{rank}\left(G_{12}\right) \leq 2 k
$$

since $k \leq n-k$. Therefore $d(\mathfrak{C}) \leq 2 k$.

Thirdly, our group $\mathbf{G}$ must be a product of cyclic groups to obtain an Abelian group. Moreover, we have chosen the generators of $\mathbf{G}$ in such a way that $\mathbf{G}$ is non-cyclic.

We finish this section recalling the well-known Lucas' Theorem [16, page 52] (see also [7, 15]) which will be useful in our construction.

Theorem 1 (Lucas' Theorem): Let b, c be nonnegative integers, $p$ a prime number and

$$
\begin{aligned}
& b=b_{0}+b_{1} p+\cdots+b_{s-1} p^{s-1}+b_{s} p^{s}, \\
& c=c_{0}+c_{1} p+\cdots+c_{s-1} p^{s-1}+c_{s} p^{s}
\end{aligned}
$$

the base $p$ expansions of $b$ and $c$ respectively. Then

$$
\left(\begin{array}{l}
b \\
c
\end{array}\right) \equiv \prod_{i=0}^{s}\left(\begin{array}{l}
b_{i} \\
c_{i}
\end{array}\right) \quad(\bmod p) .
$$


This result uses the usual convention that $\left(\begin{array}{l}0 \\ 0\end{array}\right)=1$ and $\left(\begin{array}{l}i \\ j\end{array}\right)=0$ if $i<j$.

As a consequence of Lucas' Theorem, the binomial coefficient $\left(\begin{array}{l}b \\ c\end{array}\right)$ is divisible by a prime $p$ if and only if at least one of the base $p$ digits of $c$ is greater than the corresponding digit of $b$. Therefore we obtain the following result

Corollary 1: Assume that $b, c$ and $t$ are positive integers. If $b \equiv 0\left(\bmod p^{t}\right)$ and $c<p^{t}$, then $\left(\begin{array}{l}b \\ c\end{array}\right) \equiv 0$ $(\bmod p)$.

\section{Our construction}

From now on, we will assume that $q=p^{r}$, with $r \geq 1, p$ a prime number and, $n$ and $k$ nonnegative integers such that

$$
p^{r-1} \leq n-2 k<k<n-k \leq p^{r}=q .
$$

In this section we construct an Abelian non-cyclic subgroup of $\mathrm{GL}_{n}$ which is a direct product of two cyclic subgroups of orders $p^{r}-1$ and $p^{r}\left(p^{r}-1\right)$; then, the corresponding orbit code, defined by the orbit of $\mathcal{U}_{k}$ under the action of that subgroup, has cardinality $p^{r}\left(p^{r}-1\right)$, length $n$, and the minimum distance is maximal, that is, $2 k$.

The maximum values of $k$ and $n$ which we can choose in our construction, satisfying equation (8) are $k=p^{r-1}(p-1)$ and $n=p^{r-1}(2 p-1)$. A preliminary version for $r=1$ was presented at the 5 th International Castle Meeting on Coding Theory and Applications [5].

Let $\lambda$ be a generator of the multiplicative cyclic group $\mathbb{F}_{q}^{*}$; that is, $\lambda$ is a primitive element of $\mathbb{F}_{q}^{*}$ (in particular $o(\lambda)=q-1$ is the multiplicative order of $\lambda$ ).

Let us denote by $J_{\ell} \in \mathbb{F}_{q}^{\ell \times \ell}$ the upper unitriangular matrix of size $\ell \times \ell$, with 1's on the second upper diagonal and 0's elsewhere, that is, $J_{\ell}$ is a Jordan block of size $\ell \times \ell$ associated to 1 . The following result gives the multiplicative order of $J_{\ell}$.

Lemma 2: If $p^{t-1}<\ell \leq p^{t}$ for some nonnegative integer $t$, then $o\left(J_{\ell}\right)=p^{t}$.

Proof: Let $J_{\ell}=I_{\ell}+N_{\ell}$ where

$$
N_{\ell}=\left[\begin{array}{ccccc}
0 & 1 & 0 & \cdots & 0 \\
0 & 0 & 1 & \cdots & 0 \\
\vdots & \vdots & \vdots & & \vdots \\
0 & 0 & 0 & \cdots & 1 \\
0 & 0 & 0 & \cdots & 0
\end{array}\right] \in \mathbb{F}_{q}^{\ell \times \ell}
$$

is the canonical nilpotent matrix with index $\ell$. Then $J_{\ell}^{p^{t}}=I_{\ell}^{p^{t}}+N_{\ell}^{p^{t}}=I_{\ell}$, and from the choice of $\ell$, it follows that $o\left(J_{\ell}\right)=p^{t}$.

In order to construct our subgroup $\mathbf{G}$ of $\mathrm{GL}_{n}$, we consider the following two matrices of $\mathrm{GL}_{n}$ :

$$
S=\left[\begin{array}{cc}
\lambda I_{n-k} & X \\
O & I_{k}
\end{array}\right] \quad \text { and } \quad T=\left[\begin{array}{cc}
J_{n-k} & Y \\
O & \lambda I_{k}
\end{array}\right]
$$

where $X, Y \in \mathbb{F}_{q}^{(n-k) \times k}$ are arbitrary matrices, on which we will impose additional properties as we need them to achieve our goal.

Lemma 3: For the matrices $S$ and $T$ given by expression (9), it follows that

(a) $o(S)=o(\lambda)=q-1$.

(b) $o(T)=o\left(J_{n-k}\right) o(\lambda)=q(q-1)$. 
Proof: According to Lemma 1, for any nonnegative integer $a$ we have that

$$
S^{a}=\left[\begin{array}{cc}
\lambda^{a} I_{n-k} & \Psi_{a}\left(\lambda I_{n-k}, I_{k}, X\right) \\
O & I_{k}
\end{array}\right]
$$

where

$$
\Psi_{a}\left(\lambda I_{n-k}, I_{k}, X\right)=\sum_{i=1}^{a} \lambda^{a-i} X=\frac{\lambda^{a}-1}{\lambda-1} X .
$$

Since $o(\lambda)=q-1$, we have that $\lambda^{q-1}=1$, and therefore $\Psi_{q-1}\left(\lambda I_{n-k}, I_{k}, X\right)=O$. Consequently, $o(S)=o(\lambda)=q-1$.

Let us compute the order of $T$. From Lemma 2 and our choice of $n-k$, it follows that $o\left(J_{n-k}\right)=p^{r}=q$. On the other hand, $o\left(\lambda I_{k}\right)=o(\lambda)=q-1$.

Now, by Lemma 1 for any nonnegative integer $b$ we have that

$$
T^{b}=\left[\begin{array}{cc}
J_{n-k}^{b} & \Psi_{b}\left(J_{n-k}, \lambda I_{k}, Y\right) \\
O & \lambda^{b} I_{k}
\end{array}\right]
$$

where

$$
\Psi_{b}\left(J_{n-k}, \lambda I_{k}, Y\right)=\sum_{j=1}^{b} J_{n-k}^{b-j} Y\left(\lambda I_{n-k}\right)^{j-1}=\left(\sum_{j=1}^{b} \lambda^{j-1} J_{n-k}^{b-j}\right) Y .
$$

Consequently, $o(T)=u q(q-1)$, where $u$ is the smallest nonnegative integer such that $\Psi_{u q(q-1)}\left(J_{n-k}, \lambda I_{k}, Y\right)=$ $O$. Now, following expression (3) we have that

$$
\begin{aligned}
\Psi_{t q}\left(J_{n-k}, \lambda I_{k}, Y\right) & =\sum_{\ell=1}^{t} \lambda^{t-\ell} \Psi_{q}\left(J_{n-k}, \lambda I_{k}, Y\right) \\
& =\frac{\lambda^{t}-1}{\lambda-1} \Psi_{q}\left(J_{n-k}, \lambda I_{k}, Y\right), \quad \text { for } t=1,2, \ldots
\end{aligned}
$$

In particular, $\Psi_{(q-1) q}\left(J_{n-k}, \lambda I_{k}, Y\right)=O$ because $\lambda^{q-1}=1$. Therefore $u=1$ and consequently $o(T)=$ $q(q-1)$.

As a consequence of the previous result the subgroups of $\mathrm{GL}_{n}$ generated by the matrices $S$ and $T$ are cyclic subgroups of order $q-1$ and $q(q-1)$ respectively, that is:

$$
\langle S\rangle=\left\{S^{a} \mid 0 \leq a<q-1\right\} \cong C_{q-1} \text { and }\langle T\rangle=\left\{T^{b} \mid 0 \leq b<q(q-1)\right\} \cong C_{q(q-1)} .
$$

Lemma 4: The intersection of the groups $\langle S\rangle$ and $\langle T\rangle$ is trivial; that is $\langle S\rangle \cap\langle T\rangle=\left\{I_{n}\right\}$.

Proof: Suppose that $S^{a}=T^{b}$ for some $a$ and $b$ with $0 \leq a<q-1$ and $0 \leq b<q(q-1)$; that is

$$
\left[\begin{array}{cc}
\lambda^{a} I_{n-k} & \Psi_{a}\left(\lambda I_{n-k}, I_{k}, X\right) \\
O & I_{k}
\end{array}\right]=\left[\begin{array}{cc}
J_{n-k}^{b} & \Psi_{b}\left(J_{n-k}, \lambda I_{k}, Y\right) \\
O & \lambda^{b} I_{k}
\end{array}\right]
$$

Then $\lambda^{a}=1, J_{n-k}^{b}=I_{n-k}$, and $\lambda^{b}=1$. Now, since $o(\lambda)=q-1$ and $o\left(J_{n-k}\right)=q$, the choice of $a$ and $b$ implies that $a=b=0$. Therefore by lemma $1,\langle S\rangle \cap\langle T\rangle=\left\{I_{n}\right\}$.

The following lemma, where we fix a first relation between $X$ and $Y$, is crucial for our construction. The proof is straightforward and we omit it.

Lemma 5: If $X \neq O$ and $Y=\frac{1}{\lambda-1}\left(J_{n-k}-\lambda I_{n-k}\right) X$ in expression (9), then $S T=T S$. 
The above result also holds for $X=O$, but this is a trivial case that we will not consider in the paper.

Now we are ready to present our group G. From now on, we assume that matrices $S$ and $T$ are defined by expression (9), and matrices $X$ and $Y$ are defined as in Lemma 5. Since $S T=T S$ and $\langle S\rangle \cap\langle T\rangle=\left\{I_{n}\right\}$ we obtain

$$
\begin{aligned}
\mathbf{G} & =\langle S, T\rangle=\langle S\rangle \times\langle T\rangle \\
& =\left\{S^{a} T^{b} \mid 0 \leq a<q-1,0 \leq b<q(q-1)\right\} \cong C_{q-1} \times C_{q(q-1)} ;
\end{aligned}
$$

that is, $\mathbf{G}$ is an Abelian non-cyclic group of order $q(q-1)^{2}$ and the elements of $\mathbf{G}$ present the following form

$$
\begin{aligned}
S^{a} T^{b} & =\left[\begin{array}{cc}
\lambda^{a} I_{n-k} & \Psi_{a}\left(\lambda I_{n-k}, I_{k}, X\right) \\
O & I_{k}
\end{array}\right]\left[\begin{array}{cc}
J_{n-k}^{b} & \Psi_{b}\left(J_{n-k}, \lambda I_{k}, Y\right) \\
O & \lambda^{b} I_{k}
\end{array}\right] \\
& =\left[\begin{array}{cc}
\lambda^{a} J_{n-k}^{b} & \lambda^{a} \Psi_{b}\left(J_{n-k}, \lambda I_{k}, Y\right)+\lambda^{b} \Psi_{a}\left(\lambda I_{n-k}, I_{k}, X\right) \\
O & \lambda^{b} I_{k}
\end{array}\right] .
\end{aligned}
$$

The following results will help us to understand the structure of $S^{a} T^{b}$. The first one is well known and, therefore, we omit the proof.

Lemma 6: For any nonnegative integer $b$ it follows that

$$
J_{n-k}^{b}=\left[\begin{array}{cccccc}
1 & \left(\begin{array}{c}
b \\
1
\end{array}\right) & \left(\begin{array}{c}
b \\
2
\end{array}\right) & \cdots & \left(\begin{array}{c}
b \\
n-k-2
\end{array}\right) & \left(\begin{array}{c}
b \\
b-k-1 \\
b
\end{array}\right) \\
0 & 1 & \left(\begin{array}{c}
b \\
1
\end{array}\right) & \cdots & \left(\begin{array}{c}
n-k-3 \\
b-k
\end{array}\right) & \left(\begin{array}{c}
n-k-2 \\
b
\end{array}\right) \\
0 & 0 & 1 & \cdots & \left(\begin{array}{c}
b-4 \\
n-k-4
\end{array}\right) & \left(\begin{array}{c}
n-k-3 \\
n
\end{array}\right) \\
\vdots & \vdots & \vdots & & \vdots & \vdots \\
0 & 0 & 0 & \cdots & 1 & \left(\begin{array}{c}
b \\
1
\end{array}\right) \\
0 & 0 & 0 & \cdots & 0 & 1
\end{array}\right] .
$$

Lemma 7: For matrices $X$ and $Y$ as in Lemma 5, then

$$
\lambda^{a} \Psi_{b}\left(J_{n-k}, \lambda I_{k}, Y\right)+\lambda^{b} \Psi_{a}\left(\lambda I_{n-k}, I_{k}, X\right)=\frac{\lambda^{a}}{\lambda-1}\left[\begin{array}{cccccc}
\alpha & \left(\begin{array}{c}
b \\
1
\end{array}\right) & \left(\begin{array}{c}
b \\
2
\end{array}\right) & \cdots & \left(\begin{array}{c}
b \\
n-k-2
\end{array}\right) & \left(\begin{array}{c}
b \\
n-k-1 \\
b
\end{array}\right) \\
0 & \alpha & \left(\begin{array}{c}
b \\
b
\end{array}\right) & \cdots & \left(\begin{array}{c}
n \\
b-k-3
\end{array}\right) & \left(\begin{array}{c}
n \\
n-k-2
\end{array}\right) \\
0 & 0 & \alpha & \cdots & \left(\begin{array}{c}
n \\
b-k-4
\end{array}\right) & \left(\begin{array}{c}
n-k-3 \\
n-k
\end{array}\right) \\
\vdots & \vdots & \vdots & & \vdots & \vdots \\
0 & 0 & 0 & \cdots & \alpha & \left(\begin{array}{c}
b \\
1 \\
1
\end{array}\right) \\
0 & 0 & 0 & \cdots & 0 & \alpha
\end{array}\right] X
$$

where $\alpha=\frac{\lambda^{a}-\lambda^{b}}{\lambda^{a}}$.

Proof: According to Lemmas 1 and 5, and expressions (10) and (11), we have that

$$
\begin{aligned}
\lambda^{a} \Psi_{b}\left(J_{n-k}, \lambda I_{k}, Y\right)+\lambda^{b} \Psi_{a}\left(\lambda I_{n-k}, I_{k}, X\right) & =\lambda^{a}\left(\sum_{j=1}^{b} \lambda^{j-1} J_{n-k}^{b-j}\right) Y+\lambda^{b} \frac{\lambda^{a}-1}{\lambda-1} X \\
& =\frac{\lambda^{a}}{\lambda-1} \sum_{j=1}^{b} \lambda^{j-1} J_{n-k}^{b-j}\left(J_{n-k}-\lambda I_{n-k}\right) X+\frac{\lambda^{b}\left(\lambda^{a}-1\right)}{\lambda-1} X \\
& =\frac{\lambda^{a}}{\lambda-1}\left(J_{n-k}^{b}-\lambda^{b} I_{n-k}+\frac{\lambda^{b}\left(\lambda^{a}-1\right)}{\lambda^{a}} I_{n-k}\right) X \\
& =\frac{\lambda^{a}}{\lambda-1}\left(J_{n-k}^{b}-\frac{\lambda^{b}}{\lambda^{a}} I_{n-k}\right) X
\end{aligned}
$$




$$
=\frac{\lambda^{a}}{\lambda-1}\left[\begin{array}{cccccc}
\alpha & \left(\begin{array}{c}
b \\
1
\end{array}\right) & \left(\begin{array}{c}
b \\
2
\end{array}\right) & \cdots & \left(\begin{array}{c}
b \\
n-k-2
\end{array}\right) & \left(\begin{array}{c}
b \\
n-k-1 \\
b
\end{array}\right) \\
0 & \alpha & \left(\begin{array}{c}
b \\
b
\end{array}\right) & \cdots & \left(\begin{array}{c}
n \\
b-k-3
\end{array}\right) & \left(\begin{array}{c}
n-k-2 \\
b
\end{array}\right) \\
b & 0 & \alpha & \cdots & \left(\begin{array}{c}
b \\
n-k-4
\end{array}\right) & \left(\begin{array}{c}
n-3-3 \\
n-k
\end{array}\right) \\
0 & \vdots & \vdots & & \vdots & \vdots \\
0 & 0 & 0 & \cdots & \alpha & \left(\begin{array}{c}
b \\
1
\end{array}\right) \\
0 & 0 & 0 & \cdots & 0 & \alpha
\end{array}\right] X
$$

where the last equality follows from Lemma 6 and the fact that $\alpha=1-\frac{\lambda^{b}}{\lambda^{a}}$.

Lemma 8: With the notation of Lemma 7, we have that $\alpha=0$ if, and only if, $a \equiv b(\bmod (q-1))$.

Proof: By Lemma 7, we know that $\alpha=\frac{\lambda^{a}-\lambda^{b}}{\lambda^{a}}$. Since $\lambda$ is a generator of the multiplicative cyclic group $\mathbb{F}_{q}^{*}$ the result is clear.

Note that, according to expressions (6) and (7), to define the code $\mathfrak{C}$, we lay aside the block partition of $S^{a} T^{b}$ given by expression (13); instead, we focus on the first $k$ rows of $S^{a} T^{b}$; and more specifically, on the first $k$ rows and the last $n-k$ columns of $S^{a} T^{b}$. So, we consider the following partition

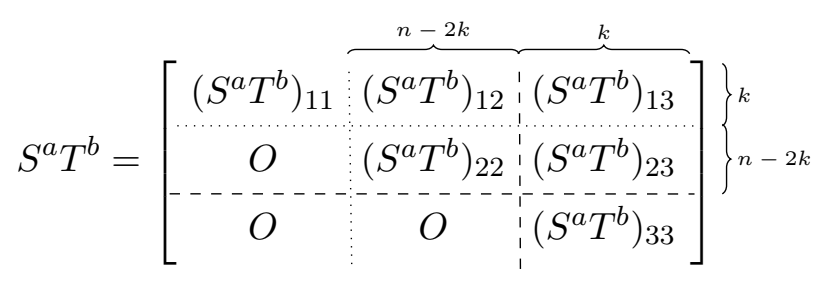

where, according to expression (13),

$$
\begin{gathered}
\lambda^{a} J_{n-k}^{b}=\left[\begin{array}{cc}
\left(S^{a} T^{b}\right)_{11} & \left(S^{a} T^{b}\right)_{12} \\
O & \left(S^{a} T^{b}\right)_{22}
\end{array}\right], \\
\lambda^{a} \Psi_{b}\left(J_{n-k}, \lambda I_{k}, Y\right)+\lambda^{b} \Psi_{a}\left(\lambda I_{n-k}, I_{k}, X\right)=\left[\begin{array}{c}
\left(S^{a} T^{b}\right)_{13} \\
\left(S^{a} T^{b}\right)_{23}
\end{array}\right], \\
\left(S^{a} T^{b}\right)_{11}=\lambda^{a} J_{k}^{b}, \quad\left(S^{a} T^{b}\right)_{22}=\lambda^{a} J_{n-2 k}^{b}, \quad\left(S^{a} T^{b}\right)_{33}=\lambda^{b} I_{k},
\end{gathered}
$$

and, $\left(S^{a} T^{b}\right)_{12}$ is the submatrix of $\lambda^{a} J_{n-k}^{b}$ formed by the first $k$ rows and the last $n-2 k$ columns; therefore, as a consequence of Lemma 6 ,

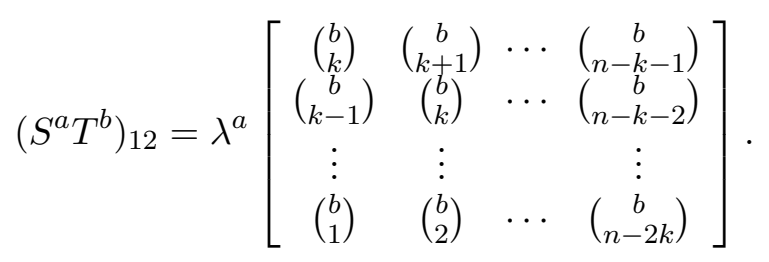

Moreover, if we assume that

$$
X=\left[\begin{array}{c}
X_{1} \\
X_{2}
\end{array}\right] \quad \text { where } X_{1} \in \mathbb{F}_{q}^{k \times k}, X_{2} \in \mathbb{F}_{q}^{(n-2 k) \times k},
$$

then, from Lemma 7, it follows that

$$
\left(S^{a} T^{b}\right)_{13}=\frac{\lambda^{a}}{\lambda-1}\left[\begin{array}{cccccc}
\alpha & \left(\begin{array}{c}
b \\
1
\end{array}\right) & \left(\begin{array}{c}
b \\
2
\end{array}\right) & \cdots & \left(\begin{array}{c}
b \\
k-2
\end{array}\right) & \left(\begin{array}{c}
b \\
k-1 \\
b
\end{array}\right) \\
0 & \alpha & \left(\begin{array}{l}
b \\
1
\end{array}\right) & \cdots & \left(\begin{array}{c}
b-3 \\
k-3
\end{array}\right) & \left(\begin{array}{c}
k-2 \\
b
\end{array}\right) \\
0 & 0 & \alpha & \cdots & \left(\begin{array}{c}
b-4 \\
k-4
\end{array}\right) & \left(\begin{array}{c}
b-3 \\
k-1
\end{array}\right) \\
\vdots & \vdots & \vdots & & \vdots & \vdots \\
0 & 0 & 0 & \cdots & \alpha & \left(\begin{array}{c}
b \\
1
\end{array}\right) \\
0 & 0 & 0 & \cdots & 0 & \alpha
\end{array}\right] X_{1}
$$




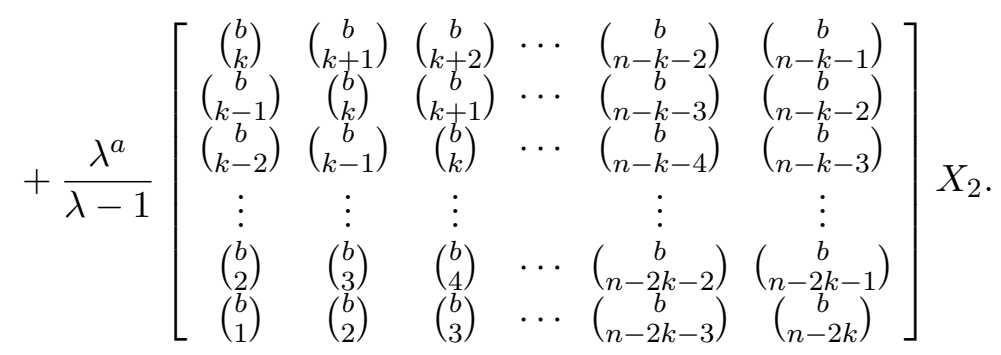

According to expression (6), the orbit code $\mathfrak{C}$ generated by the action of $\mathbf{G}$ on $\mathcal{U}_{k}$ is

$$
\begin{aligned}
\mathfrak{C} & =\left\{\mathcal{U}_{k} \cdot S^{a} T^{b} \mid 0 \leq a<q-1,0 \leq b<q(q-1)\right\} \\
& =\left\{\operatorname{rowspace}\left(\left[\left(S^{a} T^{b}\right)_{11}\left(S^{a} T^{b}\right)_{12}\left(S^{a} T^{b}\right)_{13}\right]\right) \mid 0 \leq a<q-1,0 \leq b<q(q-1)\right\} .
\end{aligned}
$$

Notice that, according to expression (7),

$$
d\left(\mathcal{U}_{k}, \mathcal{U}_{k} \cdot S^{a} T^{b}\right)=2 \operatorname{rank}\left[\left(S^{a} T^{b}\right)_{12}\left(S^{a} T^{b}\right)_{13}\right] \leq 2 k .
$$

Our next goal will be to choose $X$ in such a way that

$$
\operatorname{rank}\left[\left(S^{a} T^{b}\right)_{12}\left(S^{a} T^{b}\right)_{13}\right]=k,
$$

for all $S^{a} T^{b} \in \mathbf{G} \backslash \operatorname{Stab}_{\mathbf{G}}\left(\mathcal{U}_{k}\right)$. Consequently, $d(\mathfrak{C})=2 k$ and the minimum distance of our code will be maximal for a concrete choice of $X$.

Lemma 9: For the matrix $X$ given in (16), if $X_{1} \neq O$, then the stabilizer in $\mathbf{G}$ of $\mathcal{U}_{k}$ is the cyclic subgroup of $\mathbf{G}$ generated by $S T^{q}$; that is, $\operatorname{Stab}_{\mathbf{G}}\left(\mathcal{U}_{k}\right)=\left\langle S T^{q}\right\rangle$.

Proof: Recall that by means of expression (5) and (14) we obtain

$$
\operatorname{Stab}_{\mathbf{G}}\left(\mathcal{U}_{k}\right)=\left\{S^{a} T^{b} \in \mathbf{G} \mid\left(S^{a} T^{b}\right)_{12}=O \text { and }\left(S^{a} T^{b}\right)_{13}=O\right\} .
$$

Moreover,

$$
\left\langle S T^{q}\right\rangle=\left\{I, S T^{q}, S^{2} T^{2 q}, S^{3} T^{3 q}, \ldots, S^{q-2} T^{(q-2) q}\right\}=\left\{S^{a} T^{b} \in \mathbf{G} \mid b=a q, 0 \leq a<q-1\right\}
$$

which is a cyclic subgroup of order $q-1$.

First, we prove that $\left\langle S T^{q}\right\rangle$ is included into $\operatorname{Stab}_{\mathbf{G}}\left(\mathcal{U}_{k}\right)$.

On one hand, consider $S^{a} T^{b} \in\left\langle S T^{q}\right\rangle$, and let us see that $\left(S^{a} T^{b}\right)_{12}=O$ and $\left(S^{a} T^{b}\right)_{13}=O$. If $b=0$ then $a=0$ and $S^{a} T^{b}$ is the identity matrix. Assume that $b>0$. Since $b$ is a multiple of $q$ and $n-k \leq q$, Lucas' Theorem and Corollary 1 state that $\left(\begin{array}{l}b \\ i\end{array}\right)=0$ in $\mathbb{F}_{q}$, for all $i=1,2, \ldots, n-k-1$. It follows that $\left(S^{a} T^{b}\right)_{12}=O$, by expression (15), and that

$$
\lambda^{a} \Psi_{b}\left(J_{n-k}, \lambda I_{k}, Y\right)+\lambda^{b} \Psi_{a}\left(\lambda I_{n-k}, I_{k}, X\right)=\frac{\lambda^{a}}{\lambda-1} \alpha X
$$

by Lemma 7. Moreover, since $b=q a$, then $\alpha=0$ by Lemma 8. In particular, $\left(S^{a} T^{b}\right)_{13}=O$ and we obtain $\left\langle S T^{q}\right\rangle \subseteq \operatorname{Stab}_{\mathbf{G}}\left(\mathcal{U}_{k}\right)$.

On the other hand, if $S^{a} T^{b} \in \operatorname{Stab}_{\mathbf{G}}\left(\mathcal{U}_{k}\right)$, it follows that $\left(S^{a} T^{b}\right)_{12}=O$ and $\left(S^{a} T^{b}\right)_{13}=O$. Since $\left(S^{a} T^{b}\right)_{12}=O,\left(\begin{array}{l}b \\ i\end{array}\right)=0$ in $\mathbb{F}_{q}$, for all $i=1,2, \ldots, n-k-1$. In particular, $\left(\begin{array}{l}b \\ 1\end{array}\right)=0$ in $\mathbb{F}_{q}$. Then $b \equiv 0$ $(\bmod p)$ and we can write $b=u p^{\ell}$ where $\operatorname{gcd}(u, p)=1$ and $\ell \geq 1$. Let us see that $\ell=r$. If $\ell<r$, then $p^{\ell} \leq p^{r-1}<n-k$. Thus, $\left(\begin{array}{c}b \\ p^{\ell}\end{array}\right)=0$ in $\mathbb{F}_{q}$. However, using Lucas' Theorem, if $u=u_{0}+u_{1} p+\cdots+u_{s} p^{s}$, then $u_{0} \neq 0$ and $\left(\begin{array}{c}b \\ p^{\ell}\end{array}\right)=\left(\begin{array}{c}u_{0} \\ 1\end{array}\right) \neq 0$ in $\mathbb{F}_{q}$, a contradiction. It follows that $\ell \geq r$ and $b=u p^{\ell-r} q$. Denote 
$a_{1}=u p^{\ell-r}$. Let us see that $a=a_{1}$. First, since $0 \leq b<(q-1) q$, it follows that $0 \leq a_{1}<q-1$. This means, in particular, that $b \equiv a_{1}(\bmod (q-1))$. Moreover, by expression $(17)$ we have

$$
O=\left(S^{a} T^{b}\right)_{13}=\frac{\lambda^{a}}{\lambda-1} \alpha X_{1} .
$$

Now, since $X_{1} \neq O$, we obtain $\alpha=0$. Then, by Lemma $8, a \equiv b(\bmod (q-1))$. Therefore, $a \equiv a_{1}$ $(\bmod (q-1))$ and $a=a_{1}$, since $0 \leq a, a_{1}<q-1$. Thus, $b=a q$ and $S^{a} T^{b} \in\left\langle S T^{q}\right\rangle$.

Now, we are going to specify matrix $X$ such that the minimum distance of the code $\mathfrak{C}$ is maximal.

Theorem 2: For the matrix $X$ given in (16), if $X_{1} \in \mathrm{GL}_{k}$ and $X_{2}=O$, then

$$
\operatorname{rank}\left[\left(S^{a} T^{b}\right)_{12}\left(S^{a} T^{b}\right)_{13}\right]=k, \quad \text { for all } \quad S^{a} T^{b} \in \mathbf{G} \backslash \operatorname{Stab}_{\mathbf{G}}\left(\mathcal{U}_{k}\right) .
$$

Proof: Let $S^{a} T^{b} \in \mathbf{G} \backslash \operatorname{Stab}_{\mathbf{G}}\left(\mathcal{U}_{k}\right)$; that is, $0 \leq a<q-1,0 \leq b<q(q-1)$ and $b \neq q a$ by Lemma 9 .

By expressions (15) and (17), and the fact that

$$
\left[\begin{array}{cc}
\frac{\lambda-1}{\lambda^{a}} X_{1}^{-1} & O \\
O & \frac{1}{\lambda^{a}} I_{n-2 k}
\end{array}\right] \in \mathrm{GL}_{n-k}
$$

it follows that

$$
\begin{aligned}
& \operatorname{rank}\left[\left(S^{a} T^{b}\right)_{12}\left(S^{a} T^{b}\right)_{13}\right] \\
& =\operatorname{rank}\left[\begin{array}{ll}
\left(S^{a} T^{b}\right)_{13} & \left.\left(S^{a} T^{b}\right)_{12}\right]
\end{array}\right. \\
& =\operatorname{rank}\left(\left[\begin{array}{ll}
\left(S^{a} T^{b}\right)_{13} & \left(S^{a} T^{b}\right)_{12}
\end{array}\right]\left[\begin{array}{cc}
\frac{\lambda-1}{\lambda^{a}} X_{1}^{-1} & O \\
O & \frac{1}{\lambda^{a}} I_{n-2 k}
\end{array}\right]\right)
\end{aligned}
$$

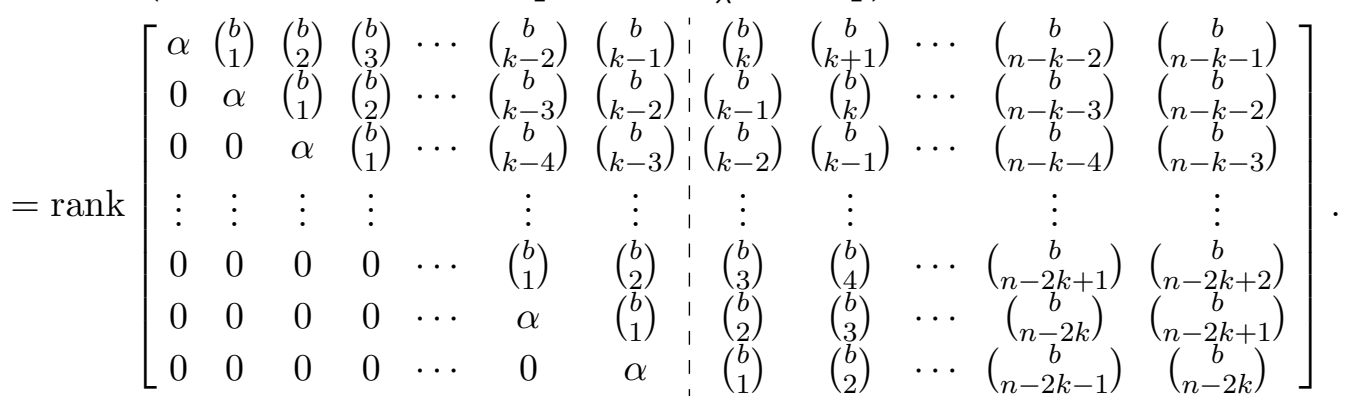

Note that if $\alpha \neq 0$, clearly $\operatorname{rank}\left[\left(S^{a} T^{b}\right)_{12}\left(S^{a} T^{b}\right)_{13}\right]=k$.

Assume now that $\alpha=0$. Then

$$
\begin{aligned}
& \operatorname{rank}\left[\begin{array}{ll}
\left(S^{a} T^{b}\right)_{12} & \left.\left(S^{a} T^{b}\right)_{13}\right]
\end{array}\right.
\end{aligned}
$$

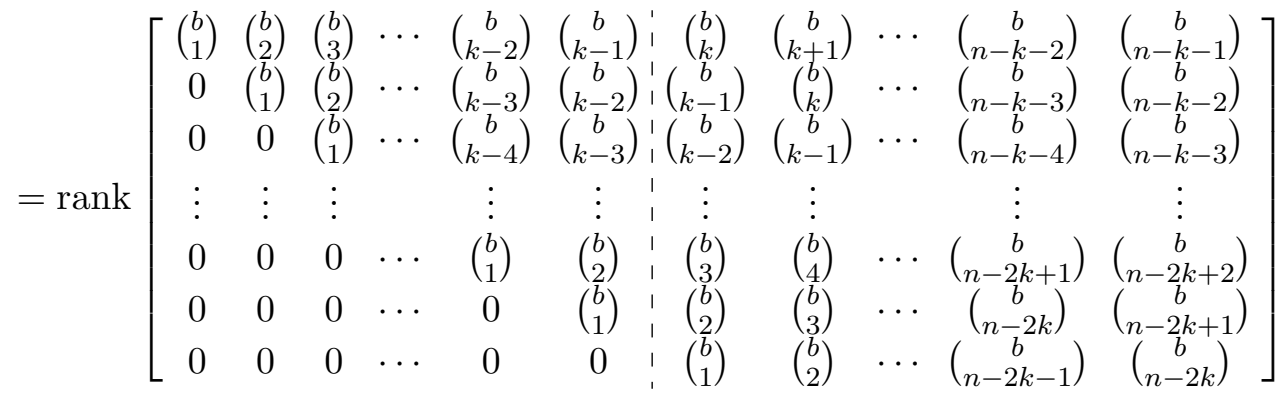

which is determined by the first nonzero element of the first row.

Assume first that $b \not \equiv 0(\bmod p)$, then $\left(\begin{array}{l}b \\ 1\end{array}\right) \neq 0$ in $\mathbb{F}_{q}$ and therefore,

$$
\operatorname{rank}\left[\left(S^{a} T^{b}\right)_{12}\left(S^{a} T^{b}\right)_{13}\right]=\min \{k, n-k-1\}=k
$$


because $k<n-k$.

Secondly, assume that $b \equiv 0(\bmod p)$. Since $b \neq \equiv 0\left(\bmod p^{r}\right)$, let $2 \leq \ell \leq r$ be such that $b \equiv 0$ $\left(\bmod p^{\ell-1}\right)$, but $b \neq \equiv 0\left(\bmod p^{\ell}\right)$; we have that $\left(\begin{array}{c}b \\ p^{\ell-1}\end{array}\right) \neq 0$ in $\mathbb{F}_{q}$ by Theorem 1 , and therefore,

$$
\operatorname{rank}\left[\left(S^{a} T^{b}\right)_{12}\left(S^{a} T^{b}\right)_{13}\right]=\min \left\{k, n-k-p^{\ell-1}\right\}=k,
$$

where the last equality follows from expression (8).

Note that for the matrix $X$ defined in the above theorem and the matrix $Y$ defined in Lemma 5, we obtain that $Y$ can be reduced to $Y=\left[\begin{array}{c}Y_{1} \\ O\end{array}\right]$ where $Y_{1}=\frac{1}{\lambda-1}\left(J_{k}-\lambda I_{k}\right) X_{1}$.

The following corollary establishes that the code $\mathfrak{C}$ defined in expression (18) is equidistant.

Corollary 2: If $\mathfrak{C}$ is the orbit code defined in expression (18), then $d\left(\mathcal{U}_{k}, \mathcal{U}_{k} \cdot G\right)=2 k$ for all $G \in$ $\mathbf{G} \backslash \operatorname{Stab}_{\mathbf{G}}(\mathcal{U})$.

Finally, as a consequence of the above results, we obtain our main theorem.

Theorem 3: Assume that $q=p^{r}$ is a prime power and let $k$ and $n$ be nonnegative integers satisfying inequalities of expression (8). Assume also that $\lambda \in \mathbb{F}_{q}$ is a primitive element. Consider the upper triangular matrices $S$ and $T$ defined in expression (9), and matrix $X$ as in Theorem 2. If $\mathbf{G}=\langle S, T\rangle$ is the Abelian non-cyclic subgroup of $\mathrm{GL}_{n}$ given in expression (12), and $\mathfrak{C}$ is the orbit code defined in expression (18), then $|\mathfrak{C}|=q(q-1)$ and $d(\mathfrak{C})=2 k$. In particular $\mathfrak{C}$ is a partial spread code.

Proof: $|\mathfrak{C}|=q(q-1)$ as a consequence of expression (12) and Lemma 9. Moreover, $d(\mathfrak{C})=2 k$ results from expressions (18), (19) and corollary 2. Finally, $\mathfrak{C}$ is a partial spread code following corollary 2 and expression (2).

The following result gives us the maximum value that the parameters $k$ and $n$ can be attained.

Theorem 4: Assume that $q=p^{r}$ is a prime power and let $k$ and $n$ be nonnegative integers satisfying the inequalities in expression (8). Then,

$$
p^{r-1}+1 \leq k \leq p^{r-1}(p-1) \quad \text { and } \quad p^{r-1}+2 k \leq n \leq p^{r}+k .
$$

Moreover, if $k=p^{r-1}(p-1)$, then $n=p^{r-1}(2 p-1)$.

Proof: From expression (8) it is an straightforward exercise to obtain the inequalities in expression (20). Moreover, if we take $k=p^{r-1}(p-1)$, it follows that $n=p^{r-1}(2 p-1)$.

Finally, as a consequence of the two previous theorems we have an orbit code which minimum distance is maximal.

Corollary 3: Assume that $q=p^{r}$ is a prime power and let $k=p^{r-1}(p-1)$ and $n=p^{r-1}(2 p-1)$. If $\mathfrak{C}$ is the orbit code defined in expression (18), then $d(\mathfrak{C})=2 p^{r-1}(p-1)$.

\section{Conclusions}

We have presented a concrete construction of an Abelian non-cyclic orbit code. For, we have used upper triangular matrices having 1 and $\lambda$ as eigenvalues, where $\lambda$ is a primitive element of the field $\mathbb{F}_{q}$. As we have shown, this code is partial spread; that is, its distance is the maximal possible, $2 k$. To our knowledge, this is the first work on Abelian non-cyclic orbit codes.

On the other hand, we have also computed its cardinality, which is, unfortunately, quite far from been optimal, since the Singleton-like bound on the size of constant dimension codes is $\frac{q^{n}-1}{q^{k}-1}$ (see [14]). This and other observations in the previous sections lead to the following open problems for future work: 
(1) Find constructions of good Abelian non-cyclic orbit codes. In other words, find a systematic way to increase the cardinality without decreasing the distance. It is our hope that the use of upper triangular matrices as generators of an Abelian non-cyclic subgroup of the general linear group, opens up new possibilities.

(2) Study the decoding of these Abelian non-cyclic orbit codes. Since we are dealing with an orbit code, we can always apply the minimum distance decoder for orbit codes proposed in [24], using a transversal of the orbits generated by $\mathbf{G}$ on the set $\mathcal{G}_{q}(k, n)$. However, despite of the general interest of this technique from a theoretical point of view, in current work we investigate if it is possible to obtain a specific good decoder exploiting the triangular form of the generator matrices of our group $\mathbf{G}$.

\section{References}

[1] R. Ahlswede, N. Cai, S.-Y. R. Li, and R. W. Yeung. Network information flow. IEEE Transactions on Information Theory, 46(4):1204-1216, 2000.

[2] F. Bardestani and A. Iranmanesh. Cyclic orbit codes with the normalizer of a Singer subgroup. Journal of Sciences, Islamic Republic of Iran, 26(1):49-55, 2015.

[3] D. Bartoli and F. Pavese. A note on equidistant subspace codes. Discrete Applied Mathematics, 198:291-296, 2016.

[4] E. Ben-Sasson, T. Etzion, A. Gabizon, and N. Raviv. Subspace polynomials and cyclic subspace codes. IEEE Transactions on Information Theory, 62(3):1157-1165, 2016.

[5] J.-J. Climent, V. Requena, and X. Soler-Escrivà. A construction of orbit codes. In Á. I. Barbero, V. Skachek, and Ø. Ytrehus, editors, Coding Theory and Applications - ICMCTA 2017, volume 10495 of Lecture Notes in Computer Science, pages 72-83. Springer International Publishing AG, Switzerland, 2017.

[6] A. Cossidente and F. Pavese. On subspace codes. Designs, Codes and Cryptography, 78(2):527-531, 2016.

[7] N. J. Fine. Binomial coefficients modulo a prime. American Mathematical Monthly, 54(10):589-592, 1947.

[8] A. Ghatak. Construction of Singer subgroup orbit codes based on cyclic difference sets. In Proceedings of the Twentieth National Conference on Communications (NCC 2014), pages 1-4, Kanpur, India, Feb. 2014. IEEE.

[9] H. Gluesing-Luerssen, K. Morrison, and C. Troha. Cyclic orbit codes and stabilizer subfields. Advances in Mathematics of Communications, 9(2):177-197, 2015.

[10] H. Gluesing-Luerssen and C. Troha. Construction of subspace codes through linkage. Advances in Mathematics of Communications, 10(3):525-540, 2016.

[11] E. Gorla and A. Ravagnani. Partial spreads in random network coding. Finite Fields and their Applications, 26:104-115, 2014.

[12] E. G. Gorla and A. Ravagnani. Equidistant subspace codes. Linear Algebra and its Applications, 490:48-65, 2016. 
[13] T. Ho, R. Koetter, M. Médard, D. R. Karger, and M. Effros. The benefits of coding over routing in a randomized setting. In Proceedings of the 2003 IEEE International Symposium on Information Theory (ISIT 2003), page 442, Yokohama, Japan, June/July 2003. IEEE.

[14] R. Kötter and F. R. Kschischang. Coding for errors and erasures in random network coding. IEEE Transactions on Information Theory, 54(8):3579-3591, 2008.

[15] A. Laugier and M. P. Saikia. A new proof of Lucas' Theorem. Proceedings of the American Mathematical Society, 18(4):1-6, 2012.

[16] É. Lucas. Sur les congruences des nombres eulériens et des coefficients différentiels des fonctions trigonométriques suivant un module premier. Bulletin de la Société Mathématique de France, 6:49$54,1878$.

[17] H. Mahdavifar and A. Vardy. Algebraic list-decoding of subspace codes. IEEE Transactions on Information Theory, 59(12):7814-7828, 2013.

[18] F. Manganiello, E. Gorla, and J. Rosenthal. Spread codes and spread decoding in network coding. In Proceedings of the 2008 IEEE International Symposium on Information Theory (ISIT 2008), pages 881-885, Toronto, Canada, July 2008. IEEE.

[19] F. Manganiello, A.-L. Trautmann, and J. Rosenthal. On conjugacy classes of subgroups of the general linear group and cyclic orbit codes. In Proceedings of the 2011 IEEE International Symposium on Information Theory (ISIT 2011), pages 1916-1920, Saint Pettersburg, July 2011. IEEE.

[20] J. Rosenthal and A.-L. Trautmann. A complete characterization of irreducible cyclic orbit codes and their Plücker embedding. Designs, Codes and Cryptography, 66:275-289, 2013.

[21] N. Silberstein and A.-L. Trautmann. New lower bounds for constant dimension codes. In Proceedings of the 2013 IEEE International Symposium on Information Theory (ISIT 2013), pages 514-518, Istanbul, July 2013. IEEE.

[22] N. Silberstein and A.-L. Trautmann. Subspace codes based on graph matchings, Ferrers diagrams, and pending blocks. IEEE Transactions on Information Theory, 61(7):3937-3953, 2015.

[23] A.-L. Trautmann. Isometry and automorphisms of constant dimension codes. Advances in Mathematics of Communications, 7(2):147-160, 2013.

[24] A.-L. Trautmann, F. Manganiello, M. Braun, and J. Rosenthal. Cyclic orbit codes. IEEE Transactions on Information Theory, 59(11):7386-7404, 2013.

[25] A.-L. Trautmann, F. Manganiello, and J. Rosenthal. Orbit codes - a new concept in the area of network coding. In Proceedings of the 2010 IEEE Information Theory Workshop (ITW 2010), Dublin, Ireland, Aug. 2010. IEEE.

[26] A.-L. Trautmann and J. Rosenthal. A complete characterization of irreducible cyclic orbit codes. In Proceedings of the Seventh International Workshop on Coding and Cryptography (WCC 2011), pages 219-228, Paris, France, Apr. 2011. 